\title{
Asymptotic Ruin Probability of a Bidimensional Risk Model Based on Entrance Processes with Constant Interest Rate
}

\author{
Hongmin Xiao and Lin Xie * \\ College of Mathematics and Statistics, Northwest Normal University, Lanzhou 730070, China; xiaohm9@126.com \\ * Correspondence: xielinmath@163.com
}

Received: 2 October 2018; Accepted: 15 November 2018; Published: 20 November 2018

check for updates

\begin{abstract}
In this paper, the risk model with constant interest based on an entrance process is investigated. Under the assumptions that the entrance process is a renewal process and the claims sizes satisfy a certain dependence structure, which belong to the different heavy-tailed distribution classes, the finite-time asymptotic estimate of the bidimensional risk model with constant interest force is obtained. Particularly, when inter-arrival times also satisfy a certain dependence structure, these formulas still hold.
\end{abstract}

Keywords: asymptotic; constant interest force; widely lower orthant dependence; ruin probability

\section{Introduction}

In this paper, we investigate a bidimensional risk model based on entrance processes, in which an insurance company operates two kinds of business. Suppose that the initial insurance fund for $i$-th class is $x_{i}$ and $S_{j}^{(i)}$ is entry time of the $j$-th policy with $0<S_{1}^{(i)}<S_{2}^{(i)}<\cdots$ and $S_{j}^{(i)}=\sum_{k=1}^{j} \theta_{k}^{(i)}$, $\theta_{1}^{(i)}=S_{1}^{(i)}, i=1,2$. The corresponding renewal process, up to the time $t$, is

$$
N_{i}(t)=\sum_{j=1}^{\infty} I_{\left\{S_{j}^{(i)} \leq t\right\}}
$$

where $I_{\{\cdot\}}$ is the indicator function.

Denote renewal function by $m_{i}(t)=E\left(N_{i}(t)\right)=\sum_{j=1}^{\infty} P\left(S_{j}^{(i)} \leq t\right), t \geq 0$ and suppose the $m_{i}(t)<\infty$ for all $0<t<\infty$ and $m_{i}(0)=0, i=1,2$. Let the validity time of the $j$-th policy be $\left\{C_{j}^{(i)}, j=1,2, \cdots\right\}$ with probability $P\left(C_{j}^{(i)}=\alpha_{\ell}\right)=p_{\ell}, \ell=1,2, \cdots, K$, and $\alpha_{1}<\alpha_{2}<\cdots<\alpha_{K}$, where they are independent and identically distributed. The premium is $f_{i}\left(C_{j}^{(i)}\right)$ and $f_{i}(\cdot)$ is a strictly increasing function. $D_{j}^{(i)}$ is claim time of the $j$-th policy and independent and identically distributed function $H_{i}(\cdot) . Y_{j}^{(i)}$ is the $j$-th claim size and identically distributed function $F_{i}(\cdot)$. Suppose that $D_{j}^{(i)}$, $Y_{j}^{(i)}$ and $C_{j}^{(i)}$ have the same distributions with random variables $D_{i}, Y_{i}$ and $C_{i}$, respectively.

Assume that an insurance company invests risk free market with force of interest $\delta>0$, then up to time $t$, the surplus process of the insurance company is written as: 


$$
\begin{aligned}
\left(\begin{array}{l}
R_{1}(t) \\
R_{2}(t)
\end{array}\right) & =\left(\begin{array}{c}
x_{1} e^{\delta t} \\
x_{2} e^{\delta t}
\end{array}\right)+\left(\begin{array}{c}
\sum_{j=1}^{N_{1}(t)} f_{1}\left(C_{j}^{(1)}\right) e^{\delta\left(t-S_{j}^{(1)}\right)} \\
\sum_{j=1}^{N_{2}(t)} f_{2}\left(C_{j}^{(2)}\right) e^{\delta\left(t-S_{j}^{(2)}\right)}
\end{array}\right) \\
& -\left(\begin{array}{c}
\sum_{j=1}^{N_{1}(t)} Y_{j}^{(1)} e^{\delta\left(t-S_{j}^{(1)}-D_{j}^{(1)}\right)} I_{\left\{S_{j}^{(1)}+D_{j}^{(1)} \leq t, D_{j}^{(1)} \leq C_{j}^{(1)}\right\}} \\
\sum_{j=1}^{N_{2}(t)} Y_{j}^{(2)} e^{\delta\left(t-S_{j}^{(2)}-D_{j}^{(2)}\right)} I_{\left\{S_{j}^{(2)}+D_{j}^{(2)} \leq t, D_{j}^{(2)} \leq C_{j}^{(2)}\right\}}
\end{array}\right) .
\end{aligned}
$$

Now the following two types of ruin times for a bidimensional risk model based on entrance processes are considered. we define the first time when both $R_{1}(t)$ and $R_{2}(t)$ become negative by

$$
\tau_{\max }\left(x_{1}, x_{2}\right)=\inf \left\{t: \max \left\{R_{1}(t), R_{2}(t)\right\}<0 \mid R_{i}(0)=x_{i}, i=1,2\right\},
$$

the first time when both $R_{1}(t)$ or $R_{2}(t)$ become negative by

$$
\tau_{\min }\left(x_{1}, x_{2}\right)=\inf \left\{t: \min \left\{R_{1}(t), R_{2}(t)\right\}<0 \mid R_{i}(0)=x_{i}, i=1,2\right\} .
$$

Then we define the corresponding ruin probabilities with the finite time $t>0$ respectively by

$$
\psi_{\max }\left(x_{1}, x_{2}, T\right)=P\left(\tau_{\max }\left(x_{1}, x_{2}\right) \leq T\right)=P\left(\bigcap_{i=1}^{2}\left\{R_{i}(s)<0\right\} \text { for some } 0 \leq s \leq T\right),
$$

and

$$
\psi_{\min }\left(x_{1}, x_{2}, T\right)=P\left(\tau_{\min }\left(x_{1}, x_{2}\right) \leq T\right)=P\left(\bigcup_{i=1}^{2}\left\{R_{i}(s)<0\right\} \text { for some } 0 \leq s \leq T\right) .
$$

We know that Li et al. (2005) put forward into a new model (LIG model) based on an entrance process and discussed asymptotic normality of the risk process. Furthermore, Some scholars got some conclusions through the study of the LIG model. Li and Kong (2007) discussed the weak convergence properties of the model. Xiao et al. (2008) studied some limit properties of the model under constant interest. Xiao and Tang (2009) studied the infinite ruin probability with constant interest within Poisson process and class $\mathcal{R}_{-\alpha}$. Xiao et al. (2013) discussed the ruin probability of LIG model. It is clear that the above literatures are improved and investigated for one-dimensional risk model based on entrance processes. Recently, people have been interested in two-dimensional risk model, see, for example, Chan et al. (2003); Li et al. (2007); Zhang and Wang (2012) and so on. It is well known that these literatures are investigated in the classical model, and the risk model based on entrance processes is more important and actual. Therefore, on the basis of above literatures, we consider a bidimensional risk model based on entrance processes.

Because Theorem 1 of Xiao and Tang (2009) is obtained under the Poisson process and the regular variation class, we know that this is far from the actual. Hence, in this paper, we consider the LIG model and obtain the finite-time ruin probability under the class $\mathcal{L} \cap \mathcal{D}$ with constant interest when claim sizes satisfy a certain dependence under the renewal process. The conclusion also extend the above Theorem 1 and Theorem 3.1 of Xiao et al. (2013). At the same time, it indicates that tail characteristics of claim distribution determine the ruin probability of insurance company, which is of great significance to the safe operation and the risk assessment of insurance company.

This paper is organized as follows: The second Section introduces the preliminary knowledge. The third Section presents the main results of this paper. The fourth Section gives some lemmas. Finally, the fifth Section gives the proofs of main Theorems. 


\section{Some Preliminaries}

Firstly, we give some markers. All limit relationships of this paper are for $x \rightarrow \infty$ unless stated otherwise. For the two positive function $f(\cdot)$ and $g(\cdot)$, if limsup $\frac{f(x)}{g(x)}<\infty$, write $f(x)=O(g(x))$; if $\lim \frac{f(x)}{g(x)}=0$, write $f(x)=o(g(x))$; if $\limsup \frac{f(x)}{g(x)} \leq 1$, write $f(x) \lesssim g(x)$; if $\liminf \frac{f(x)}{g(x)} \geq 1$, write $f(x) \gtrsim g(x)$; if $\lim \frac{f(x)}{g(x)}=1$, write $f(x) \sim g(x)$; if $f(x)=O(g(x)), g(x)=O(f(x))$, write $f(x) \asymp g(x)$.

Here are some important concepts of heavy-tailed distributions.

Definition 1. Say a distribution $F$ belongs to the class $\mathcal{L}$, if $F$ satisfies for any $y>0$ (or equivalent for $y=1$ )

$$
\lim _{x \rightarrow \infty} \frac{\bar{F}(x+y)}{\bar{F}(x)}=1
$$

Say a distribution $F$ belongs to the class $\mathcal{D}$, if $F$ satisfies for any $0<y<1$ (or equivalent for $y=\frac{1}{2}$ )

$$
\limsup _{x \rightarrow \infty} \frac{\bar{F}(x y)}{\bar{F}(x)}<\infty
$$

Say a distribution $F$ belongs to the class $\mathcal{C}$, if $F$ satisfies

$$
\lim _{y \uparrow 1} \limsup _{x \rightarrow \infty} \frac{\bar{F}(x y)}{\bar{F}(x)}=1 .
$$

where their relationship is as follows:

$$
\mathcal{C} \subset \mathcal{L} \cap \mathcal{D} \subset \mathcal{L}
$$

For more properties and applications of the heavy-tailed distribution, we can refer to Bingham et al. (1987) and Embrechts et al. (1997).

There are an important relationship between heavy-tailed distribution and Matuszewska index of the distribution, which is defined by

$$
J_{F}^{+}=-\lim _{x \rightarrow \infty} \frac{\log \bar{F}_{*}(x)}{\log x}, J_{F}^{-}=-\lim _{x \rightarrow \infty} \frac{\log \bar{F}^{*}(x)}{\log x}
$$

where

$$
\bar{F}_{*}(y)=\liminf _{x \rightarrow \infty} \frac{\bar{F}(x y)}{\bar{F}(x)}, \bar{F}^{*}(y)=\limsup _{x \rightarrow \infty} \frac{\bar{F}(x y)}{\bar{F}(x)} .
$$

Furthermore, other indices of the distribution $F$ can be defined by

$$
L_{F}=\lim _{y \downarrow 1} \bar{F}_{*}(y) .
$$

As for any $y>0$, there is $\bar{F}^{*}(y)=1 / \bar{F}_{*}(y)$, hence

$$
L_{F}=\lim _{y \downarrow 1} \bar{F}_{*}(y)=1 / \lim _{y \uparrow 1} \bar{F}^{*}(y) .
$$

Particularly, if $F \in \mathcal{C}$, then $L_{F}=1$.

For more properties and applications of the heavy tailed distribution, we can refer to Tang and Tsitsiashvili (2003) and Yang and Wang (2010).

Here we introduce some concepts and properties of dependence. 
Definition 2. If there exists the finite real sequence $\left\{g_{U}(n), n \geq 1\right\}$ for $x_{i} \in(-\infty, \infty), 1 \leq i \leq n$ such that

$$
P\left(\bigcap_{i=1}^{n}\left\{\xi_{i}>x_{i}\right\}\right) \leq \mathrm{g}_{U}(n) \prod_{i=1}^{n} P\left(\xi_{i}>x_{i}\right),
$$

then we say random variable sequence $\left\{\xi_{n}, n \geq 1\right\}$ are widely upper orthant dependent (WUOD).

If there exists the finite real sequence $\left\{\mathrm{g}_{L}(n), n \geq 1\right\}$ for $x_{i} \in(-\infty, \infty), 1 \leq i \leq n$ such that

$$
P\left(\bigcap_{i=1}^{n}\left\{\xi_{i} \leq x_{i}\right\}\right) \leq \mathrm{g}_{L}(n) \prod_{i=1}^{n} P\left(\xi_{i} \leq x_{i}\right)
$$

then we say random variable sequence $\left\{\xi_{n}, n \geq 1\right\}$ are widely lower orthant dependent (WLOD).

Furthermore, if $\left\{\xi_{n}, n \geq 1\right\}$ satisfy WUOD and WLOD at the same time, then we say random variables $\left\{\xi_{n}, n \geq 1\right\}$ are widely orthant dependent (WOD).

For more detailed information, we can refer to Wang et al. (2013); Ghosh (1981) and Block et al. (1982).

Definition 3. If real valued random variables $X_{i}, i \geq 1$ with distribution functions $F_{i}, i \geq 1$ satisfy for any $i \neq j$

$$
\lim _{x \rightarrow \infty} P\left(\left|X_{i}\right| \wedge X_{j}>x \mid X_{i} \vee X_{j}>x\right)=0,
$$

or, equivalently

$$
\lim _{x \rightarrow \infty} \frac{P\left(X_{i}>x, X_{j}>x\right)+P\left(X_{i}<-x, X_{j}>x\right)}{\bar{F}_{i}(x)+\bar{F}_{j}(x)}=0,
$$

then we say random variable variables $X_{i}, i \geq 1$ are pairwise quasi-asymptotically independent (PQAI).

If real valued random variables $X_{i}, i \geq 1$ with distribution functions $F_{i}, i \geq 1$ satisfy for any $i \neq j$

$$
\lim _{x_{i} \wedge x_{j} \rightarrow \infty} P\left(\left|X_{i}\right|>x \mid X_{j}>x\right)=0,
$$

or, equivalently

$$
\lim _{x_{i} \wedge x_{j} \rightarrow \infty} \frac{P\left(X_{i}>x, X_{j}>x\right)+P\left(X_{i}<-x, X_{j}>x\right)}{\bar{F}_{j}(x)}=0,
$$

then we say random variable variables $X_{i}, i \geq 1$ are pairwise strong quasi-asymptotically independent (PSQAI).

Remark 1. If random variables $X_{i}, i \geq 1$ are PSQAI, then they are PQAI.

For more detailed information, we can refer to $\mathrm{Li}$ (2013) and Liu et al. (2012).

The first lemma comes from Theorem 2.1 of Li (2013).

Lemma 1. Assume that $\left\{X_{j}, 1 \leq j \leq n\right\}$ are $n$ real-valued random variables with functions of distribution $F_{j}, 1 \leq j \leq n$. Then

$$
P\left(\sum_{j=1}^{n} c_{j} X_{j}>x\right) \sim \sum_{j=1}^{n} P\left(c_{j} X_{j}>x\right) .
$$

holds if either (i) $\left\{X_{j}\right\}$ are PSQAI and $F_{j} \in \mathcal{L} \cap \mathcal{D}$ for $1 \leq j \leq n$ and $\left(c_{1}, \cdots, c_{n}\right) \in[a, b]^{n}$, or (ii) $\left\{X_{j}\right\}$ are $P Q A I$ and $F_{j} \in \mathcal{C}$ for $1 \leq j \leq n$ and $\left(c_{1}, \cdots, c_{n}\right) \in[a, b]^{n}$.

The following lemma comes from Proposition 2.2.1 of Bingham et al. (1987). 
Lemma 2. Let $F \in \mathcal{D}$. For any $0<\alpha^{\prime}<\alpha<\beta<\beta^{\prime}$, there exist positive constants $A_{i}$ and $B_{i}, i=1,2$ satisfying the following inequality

$$
\frac{\bar{F}(y)}{\bar{F}(x)} \geq A_{1}(x / y)^{\alpha^{\prime}}
$$

for any $x \geq y \geq B_{1}$, and the inequality

$$
\frac{\bar{F}(y)}{\bar{F}(x)} \leq A_{2}(x / y)^{\beta^{\prime}}
$$

for any $x \geq y \geq B_{2}$.

The following lemma comes from Theorem 3.3 of Cline and Samorodnitsky (1994), Lemma 3.4 of Liu and Wang (2016) and Lemma 3.5 of Tang and Tsitsiashvili (2003).

Lemma 3. Let $X$ be a random variable with distribution $F$ and $Y$ be a random variable independent of $X$. Suppose that $H$ is the distribution of XY. If $E Y^{p}<\infty$ for any $0<J_{F}^{+} \leq \beta<\beta^{\prime}$ and some $p>J_{F}^{+}$, then there exist the following conclusions:

(i) If $F \in \mathcal{D}$, then $\bar{F}(x) \asymp \bar{H}(x)$ and $x^{-\beta^{\prime}}=o(\bar{F}(x))$;

(ii) If $F \in \mathcal{C}, H \in \mathcal{C}$.

The following lemma can be proved in Appendix A.

Lemma 4. (1) Under the conditions of Theorem 1 (or Theorem 2), for all $T \geq T_{0}$, then we have

$$
\begin{aligned}
& P\left(\sum_{j=1}^{\infty} Y_{j}^{(i)} e^{-\delta\left(S_{j}^{(i)}+D_{j}^{(i)}\right)} I_{\left\{S_{j}^{(i)}+D_{j}^{(i)} \leq T, D_{j}^{(i)} \leq C_{j}^{(i)}\right\}} I_{\left\{S_{j}^{(i)} \leq T\right\}}>x_{i}\right) \\
\sim & \sum_{j=1}^{\infty} P\left(Y_{j}^{(i)} e^{-\delta\left(S_{j}^{(i)}+D_{j}^{(i)}\right)} I_{\left\{S_{j}^{(i)}+D_{j}^{(i)} \leq T, D_{j}^{(i)} \leq C_{j}^{(i)}\right\}} I_{\left\{S_{j}^{(i)} \leq T\right\}}>x_{i}\right) .
\end{aligned}
$$

(2) Suppose the conditions of Theorem 2 are true and the inter-arrival times $\left\{\theta_{j}^{i}, j \geq 1\right\}$ are WLOD random variables satisfying (16) for some $\epsilon_{i 0}>0$, depending on $F_{i}$ and $G_{i}, i=1,2$. Then, for all $T \geq T_{0}$, the relation (6) still holds uniformly.

\section{Main Results}

In this paper, we make the following assumptions:

$A_{1}$ Assume that the $i$-th class of random variables $\left\{C_{j}^{(i)}, j \geq 1\right\},\left\{D_{j}^{(i)}, j \geq 1\right\},\left\{Y_{j}^{(i)}, j \geq 1\right\}$, $\left\{S_{j}^{(i)}, j \geq 1\right\}, i=1,2$, are independent mutually.

$A_{2}$ Assume that $E\left(N_{i}(t)\right)^{p+1}<\infty$ for any fixed $t>0$ and some $p>J_{F_{i}}^{+}, i=1,2$.

Theorem 1. Consider the bidimensional risk model (1) under the assumptions $A_{1}-A_{2}$. Let $p_{0}=P\left(S_{1}^{(i)}<T_{0}\right)>0$ for some $0<T_{0}<\infty$. Assume that claim sizes, $\left\{Y_{j}^{(i)}, j \geq 1\right\}$ be PSQAI random variables with common distribution $F_{i} \in \mathcal{L} \cap \mathcal{D}, i=1,2$ such that $J_{F_{i}}^{-}>0$, respectively. Then for $T \geq T_{0}$, we have

$$
\psi_{\max }\left(x_{1}, x_{2}, T\right) \sim \prod_{i=1}^{2}\left(\sum_{\ell=1}^{K} p_{\ell} \int_{0}^{T} \int_{0}^{\alpha_{\ell} \wedge(T-s)} \bar{F}_{i}\left(e^{\delta(s+y)} x_{i}\right) d H_{i}(y) d m_{i}(s)\right) .
$$


and

$$
\psi_{\min }\left(x_{1}, x_{2}, T\right) \sim \sum_{i=1}^{2} \sum_{\ell=1}^{K} p_{\ell} \int_{0}^{T} \int_{0}^{\alpha_{\ell} \wedge(T-s)} \bar{F}_{i}\left(e^{\delta(s+y)} x_{i}\right) d H_{i}(y) d m_{i}(s)
$$

where $a \wedge b=\min \{a, b\}$.

Proof. (i) Firstly, we deal with the relation (7). Write $U_{i}(t)=\sum_{j=1}^{N_{i}(t)} Y_{j}^{(i)} e^{-\delta\left(S_{j}^{(i)}+D_{j}^{(i)}\right)}$ $I_{\left\{S_{j}^{(i)}+D_{j}^{(i)} \leq t, D_{j}^{(i)} \leq C_{j}^{(i)}\right\}}$. Due to (1) and (2), we know that for all $T \geq T_{0}$,

$$
\psi_{\max }\left(x_{1}, x_{2}, T\right)=P\left(\bigcap_{i=1}^{2}\left\{U_{i}(s)-\sum_{j=1}^{N_{i}(t)} f_{i}\left(C_{j}^{(i)}\right) e^{-\delta S_{j}^{(i)}}>x_{i}\right\} \text { for some } 0 \leq s \leq T\right) .
$$

By (9), we know for all $T \geq T_{0}$,

$$
P\left(\bigcap_{i=1}^{2}\left\{U_{i}(T)-\sum_{j=1}^{N_{i}(T)} f_{i}\left(C_{j}^{(i)}\right) e^{-\delta S_{j}^{(i)}}>x_{i}\right\}\right) \leq \psi_{\max }\left(x_{1}, x_{2}, T\right) \leq P\left(\bigcap_{i=1}^{2}\left\{U_{i}(T)>x_{i}\right\}\right) .
$$

Because Lemma 4 (1) and random variables $\left\{C_{j}^{(i)}, j \geq 1\right\},\left\{D_{j}^{(i)}, j \geq 1\right\},\left\{Y_{j}^{(i)}, j \geq 1\right\},\left\{S_{j}^{(i)}, j \geq 1\right\}$, $i=1,2$, are independent mutually, it is clear that

$$
\begin{aligned}
\psi_{\max }\left(x_{1}, x_{2}, T\right) & \leq \prod_{i=1}^{2} P\left(U_{i}(T)>x_{i}\right) \\
& \sim \prod_{i=1}^{2} P\left(\sum_{j=1}^{\infty} Y_{j}^{(i)} e^{-\delta\left(S_{j}^{(i)}+D_{j}^{(i)}\right)} I_{\left\{S_{j}^{(i)}+D_{j}^{(i)} \leq T, D_{j}^{(i)} \leq C_{j}^{(i)}\right\}} I_{\left\{S_{j}^{(i)} \leq T\right\}}>x_{i}\right) \\
& \sim \prod_{i=1}^{2}\left(\sum_{\ell=1}^{K} p_{\ell} \int_{0}^{T} \int_{0}^{\alpha_{\ell} \wedge(T-s)} \bar{F}_{i}\left(e^{\delta(s+y)} x_{i}\right) d H_{i}(y) d m_{i}(s)\right) .
\end{aligned}
$$

holds uniformly for all $T \geq T_{0}$.

Because $f_{i}\left(C_{j}^{(i)}\right), j=1, \cdots, K$ are bounded and $\left\{Y_{j}^{(i)}, j \geq 1\right\}$ are PSQAI random variables with distributions $F_{i} \in \mathcal{L} \cap \mathcal{D}, i=1,2$, respectively, it is easy to prove that $\left\{Y_{j}^{(i)}+f_{i}\left(C_{j}^{(i)}\right), j \geq 1\right\}$ are PSQAI random variables, whose distributions belong to the class $\mathcal{L} \cap \mathcal{D}, i=1,2$, respectively. Hence, by Lemma 4 (1), we know

$$
\begin{aligned}
\psi_{\max }\left(x_{1}, x_{2}, T\right) & \geq P\left(\prod_{i=1}^{2}\left\{U_{i}(T)-\sum_{j=1}^{N_{i}(T)} f_{i}\left(C_{j}^{(i)}\right) e^{-\delta S_{j}^{(i)}}>x_{i}\right\}\right) \\
& =\prod_{i=1}^{2} P\left(\left\{U_{i}(T)-\sum_{j i}^{N_{i}(T)} f_{i}\left(C_{j}^{(i)}\right) e^{-\delta S_{j}^{(i)}}>x_{i}\right\}\right) \\
& =\prod_{i=1}^{2} P\left(\sum_{j=1}^{N_{i}(t)}\left(Y_{j}^{(i)} e^{-\delta\left(S_{j}^{(i)}+D_{j}^{(i)}\right)} I_{\left\{S_{j}^{(i)}+D_{j}^{(i)} \leq t, D_{j}^{(i)} \leq C_{j}^{(i)}\right\}}-f_{i}\left(C_{j}^{(i)}\right) e^{-\delta S_{j}^{(i)}}\right)>x_{i}\right) \\
& \sim \prod_{i=1}^{2} P\left(\sum_{j=1}^{\infty} Y_{j}^{(i)} e^{-\delta\left(S_{j}^{(i)}+D_{j}^{(i)}\right)} I_{\left\{S_{j}^{(i)}+D_{j}^{(i)} \leq t, D_{j}^{(i)} \leq C_{j}^{(i)}\right\}} I_{\left\{S_{j}^{(i)} \leq t\right\}}>x_{i}\right) \\
& \sim \prod_{i=1}^{2}\left(\sum_{\ell=1}^{K} p_{\ell} \int_{0}^{T} \int_{0}^{\alpha_{\ell} \wedge(T-s)} \bar{F}_{i}\left(e^{\delta(s+y)} x_{i}\right) d H_{i}(y) d m_{i}(s)\right) .
\end{aligned}
$$

Combining (11) with (12), we obtain that (7) holds uniformly for all $T \geq T_{0}$.

Next, we handle (8). By (3), we know that

$$
\psi_{\min }\left(x_{1}, x_{2}, T\right)=P\left(\tau_{1}\left(x_{1} \leq T\right)\right)+P\left(\tau_{2}\left(x_{2} \leq T\right)\right)-\psi_{\max }\left(x_{1}, x_{2}, T\right) .
$$

where $\left(\tau_{i}\left(x_{i}\right)=\inf \left\{t: R_{i}(t)<0 \mid R_{i}(0)=x_{i}\right\}, i=1\right.$, 2. Then by (7) with its one-dimensional case, it is clear that

$$
P\left(\tau_{i}\left(x_{i}\right) \leq T\right) \sim \sum_{\ell=1}^{K} p_{\ell} \int_{0}^{T} \int_{0}^{\alpha_{\ell} \wedge(T-s)} \bar{F}_{i}\left(e^{\delta(s+y)} x_{i}\right) d H_{i}(y) d m_{i}(s), \quad i=1,2 .
$$


holds uniformly for all $T \geq T_{0}$.

Again by (7) and $F_{i} \in \mathcal{L} \cap \mathcal{D} \subset \mathcal{D}, i=1,2$, we have that

$$
\begin{aligned}
& \limsup \sup _{x_{1} \wedge x_{2} \rightarrow \infty} \sup _{T \in \Lambda \cap\left[T_{0}, \infty\right)} \frac{\psi_{\max }\left(x_{1}, x_{2}, T\right)}{\sum_{i=1}^{2} \sum_{\ell=1}^{K} p_{\ell} \int_{0}^{T} \int_{0}^{\alpha_{\ell} \wedge(T-s)} \bar{F}_{i}\left(e^{\delta(s+y)} x_{i}\right) d H_{i}(y) d m_{i} s} \\
& \leq \lim \sup _{x_{1} \wedge x_{2} \rightarrow \infty} \sup _{T \in \Lambda \cap\left[T_{0}, \infty\right)} \frac{\bar{F}_{1}\left(x_{1}\right) m_{1}(T) \bar{F}_{2}\left(x_{2}\right) m_{2}(T)\left(\sum_{\ell=1}^{K} p_{\ell} H_{i}\left(\alpha_{\ell} \wedge(T-s)\right)\right)^{2}}{\bar{F}_{1}\left(x_{1} e^{\delta T}\right) m_{1}\left(T_{0}\right)\left(\sum_{\ell=1}^{K} p_{\ell} H_{i}\left(\alpha_{\ell} \wedge\left(T_{0}-s\right)\right)\right)}
\end{aligned}
$$

Hence, by (13)-(15), we prove that (8) holds uniformly for all $T \geq T_{0}$.

Theorem 2. Consider the bidimensional risk model (1) under the assumption $A_{1}-A_{2}$. Let $p_{0}=P\left(S_{1}^{(i)}<\right.$ $\left.T_{0}\right)>0$ for some $0<T_{0}<\infty$. Assume that claim sizes, $\left\{Y_{i}, i \geq 1\right\}$ be PQAI random variables with common distribution $F_{i} \in \mathcal{C}, i=1,2$ such that $J_{F_{i}}^{-}>0$, respectively. Then the relations (7) and (8) hold uniformly for $T \geq T_{0}$.

Proof. Similarly, when $f_{i}\left(C_{j}^{(i)}\right), j=1, \cdots, K$ are bounded and $\left\{Y_{j}^{(i)}, j \geq 1\right\}$ are PQAI random variables with distributions $F_{i} \in \mathcal{C}, i=1,2$, respectively, it is easy to prove that $\left\{Y_{j}^{(i)}+f_{i}\left(C_{j}^{(i)}\right), j \geq 1\right\}$ are $P Q A I$ random variables, whose distributions belong to the class $\mathcal{C}, i=1,2$, respectively. Hence, applying the same method of proof of Theorem 1, we know that (7) and (8) still hold uniformly for all $T \geq T_{0}$.

Theorem 3. Under the conditions of Theorem 2, suppose that entry inter-arrival times, $\left\{\theta_{k}^{(i)}, k \geq 1\right\}$ are $W L O D$ random variables with common distribution $G_{i}$ satisfying

$$
\lim _{n \rightarrow \infty} g_{L_{i}}(n) e^{-\epsilon_{i 0} n}=0 .
$$

holds for some $\epsilon_{i 0}>0$, depending on $F_{i}$ and $G_{i}, i=1,2$. Then, the relations (7) and (8) still hold uniformly for all $T \geq T_{0}$.

Proof. By the Lemma 4 (2), Theorem 2 and similar proof of Theorem 1, the relations (7) and (8) still hold uniformly for all $T \geq T_{0}$.

Corollary 1. Consider one-dimensional risk model satisfying the same conditions as those in Theorem 1, and denote ruin time by $\tau(x)=\inf \{t: R(t)<0 \mid R(0)=x\}$, where

$$
R(t)=x e^{\delta t}+\sum_{j=1}^{N(t)} f\left(C_{j}\right) e^{\delta\left(t-S_{j}\right)}-\sum_{j=1}^{N(t)} Y_{j} e^{\delta\left(t-S_{j}-D_{j}\right)} I_{\left\{S_{j}+D_{j} \leq t, D_{j} \leq C_{j}\right\}},
$$

then, we have

$$
\psi(x, T)=P(\tau(x) \leq T) \sim \sum_{\ell=1}^{K} p_{\ell} \int_{0}^{T} \int_{0}^{\alpha_{\ell} \wedge(T-s)} \bar{F}\left(e^{\delta(s+y)} x\right) d H(y) d m(s)
$$

It is easy to prove Corollary 1 from the proof of relation (7) of Theorem 1.

Remark 2. Corollary 1 is a partial extension for the results of Theorem 3.1 of Xiao et al. (2013), Theorem 1 of Xiao and Tang (2009), and Theorem 3.1 of Xiao and Xie (2018).

\section{Conclusions}

In summary, this paper studies the two-dimensional independent risk model based on entrance processes with constant interest rate. Under the assumptions that the entry process of policies of two kinds of business of insurance companies have different renewal processes, the claims sizes of two 
kinds of business are independent of each other, and the claims sizes of the same kind of business are pairwise strong quasi-asymptotically independent, which belong to the class $\mathcal{L} \cap \mathcal{D}$, the maximum finite-time ruin probability and the minimum finite-time ruin probability are obtained, respectively. If intervals of entry time of the policy satisfy the wide lower quadrant dependence, The finite-time maximum ruin probability and the finite-time minimum ruin probability are also obtained.

Author Contributions: L.X. completed the article preliminarily, and the Professor H.X. perfected it.

Funding: This research was funded by Natural Scientific Funds of China (71261023).

Conflicts of Interest: The authors declare no conflicts of interest.

\section{Appendix A. Proof of Lemma 4}

Proof. (1) Firstly, we deal with the upper bound of (6). Along with the method of proof of Theorem 2.1 of Hao and Tang (2008), for any positive integer $N$, we have

$$
\begin{aligned}
& P\left(\sum_{j=1}^{\infty} Y_{j}^{(i)} e^{-\delta\left(S_{j}^{(i)}+D_{j}^{(i)}\right)} I_{\left\{S_{S}^{(i)}+D_{j}^{(i)} \leq T, D_{j}^{(i)} \leq C_{j}^{(i)}\right\}} I_{\left\{S_{j}^{(i)} \leq T\right\}}>x_{i}\right) \\
& =P\left(\sum_{j=1}^{N_{i}(T)} Y_{j}^{(i)} e^{-\delta\left(S_{j}^{(i)}+D_{j}^{(i)}\right)} I_{\left\{S_{j}^{(i)}+D_{j}^{(i)} \leq T, D_{j}^{(i)} \leq C_{j}^{(i)}\right\}}>x_{i}\right) \\
& =\left(\sum_{n=1}^{N}+\sum_{n=N+1}^{\infty}\right) P\left(\sum_{j=1}^{n} Y_{j}^{(i)} e^{-\delta\left(S_{j}^{(i)}+D_{j}^{(i)}\right)} I_{\left\{S_{j}^{(i)}+D_{j}^{(i)} \leq T, D_{j}^{(i)} \leq C_{j}^{(i)}\right\}}>x_{i}, N_{i}(T)=n\right) \\
& =I_{1}\left(x_{i}, t, N\right)+I_{2}\left(x_{i}, t, N\right) .
\end{aligned}
$$

For $I_{2}\left(x_{i}, t, N\right)$. Because of the Lemma 2, there exist positive constants $\beta, A_{1}$ and $B_{1}$ for any $h>0$ and $\frac{x_{i}}{n} \geq B_{1}$. Hence, we have

$$
\begin{aligned}
I_{2}\left(x_{i}, t, N\right) & \leq \sum_{n=N+1}^{\infty} P\left(\sum_{j=1}^{n} Y_{j}^{(i)} e^{-\delta\left(S_{1}^{(i)}+D_{j}^{(i)}\right)} I_{\left\{S_{1}^{(i)}+D_{j}^{(i)} \leq T, D_{j}^{(i)} \leq C_{j}^{(i)}\right\}}>x_{i}, S_{n}^{(i)} \leq T \leq S_{n+1}^{(i)}\right) \\
& =\sum_{n=N+1}^{\infty} \int_{0}^{T} P\left(\sum_{j=1}^{n} Y_{j}^{(i)} e^{-\delta\left(s+D_{j}^{(i)}\right)} I_{\left\{s+D_{j}^{(i)} \leq T, D_{j}^{(i)} \leq C_{j}^{(i)}\right\}}>x_{i}, S_{n}^{(i)}-S_{1}^{(i)} \leq T-s \leq S_{n+1}^{(i)}-S_{1}^{(i)}\right) \\
& \cdot P\left(S_{1}^{(i)} \in d s\right) \\
& \leq \sum_{n=N+1}^{\infty} \int_{0}^{T} P\left(\sum_{j=1}^{n+1} Y_{j}^{(i)} e^{-\delta\left(s+D_{j}^{(i)}\right)} I_{\left\{s+D_{j}^{(i)} \leq T, D_{j}^{(i)} \leq C_{j}^{(i)}\right\}}>x_{i}\right) \cdot P\left(N_{i}(T-s)=n\right) P\left(S_{1}^{(i)} \in d s\right) \\
& =\sum_{N \leq n \leq x_{i} / B_{1}} \int_{0}^{T} P\left(\sum_{j=1}^{n+1} Y_{j}^{(i)} e^{-\delta\left(s+D_{j}^{(i)}\right)} I_{\left\{s+D_{j}^{(i)} \leq T, D_{j}^{(i)} \leq C_{j}^{(i)}\right\}}>x_{i}\right) P\left(N_{i}(T-s)=n\right) d m_{i}(s) \\
& +\sum_{n>x_{i} / B_{1}} \int_{0}^{T} P\left(\sum_{j=1}^{n+1} Y_{j}^{(i)} e^{-\delta\left(s+D_{j}^{(i)}\right)} I_{\left\{s+D_{j}^{(i)} \leq T, D_{j}^{(i)} \leq C_{j}^{(i)}\right\}}>x_{i}\right) P\left(N_{i}(T-s)=n\right) d m_{i}(s) \\
& \leq \sum_{N \leq n \leq x_{i} / B_{1}} \int_{0}^{T}(n+1) P\left(Y_{j}^{(i)} e^{-\delta\left(s+D_{j}^{(i)}\right)} I_{\left\{s+D_{j}^{(i)} \leq T, D_{j}^{(i)} \leq C_{j}^{(i)}\right\}}>x_{i} /(n+1)\right) P\left(N_{i}(T-s)=n\right) \\
& \cdot d m_{i}(s)+\sum_{n>x_{i} / B_{1}} \int_{0}^{T} P\left(N_{i}(T-s)=n\right) d m_{i}(s) \\
& \leq \sum_{N \leq n \leq x_{i} / B_{1}} \int_{0}^{T} C_{1} P\left(Y_{i} e^{-\delta\left(s+D_{i}\right)} I_{\left\{s+D_{i} \leq T, D_{i} \leq C_{i}\right\}}>x_{i}\right)(n+1)^{\beta+1} P\left(N_{i}(T-s)=n\right) d m_{i}(s) \\
& +\int_{0}^{T} P\left(N_{i}(T-s)>x_{i} / B_{1}\right) d m_{i}(s)
\end{aligned}
$$




$$
\begin{aligned}
& \leq A_{1} \int_{0}^{T} P\left(Y_{i} e^{-\delta\left(s+D_{i}\right)} I_{\left\{s+D_{i} \leq T, D_{i} \leq C_{i}\right\}}>x_{i}\right) E\left(N_{i}(T-s)+1\right)^{\beta+1} I_{\left\{N \leq N_{0}(T-s) \leq x_{i} / B_{1}\right\}} d m_{i}(s) \\
& +P\left(N_{i}(T-s)>x_{i} / B_{1}\right) m_{i}(T) \\
& \leq A_{1} E\left(N_{i}(T-s)+1\right)^{\beta+1} I_{\left\{N \leq N_{0}(T-s) \leq x_{i} / B_{1}\right\}} \int_{0}^{T} P\left(Y_{i} e^{-\delta\left(s+D_{i}\right)} I_{\left\{s+D_{i} \leq T, D_{i} \leq C_{i}\right\}}>x_{i}\right) d m_{i}(s) \\
& +e^{-h\left(x_{i} / B_{1}\right)} E\left(e^{h N_{i}(T)}\right) m_{i}(T) .
\end{aligned}
$$

If $N \rightarrow \infty$, then we have

$$
E\left(N_{i}(T-s)+1\right)^{\beta+1} I_{\left\{N \leq N_{i}(T-s) \leq x_{i} / B_{1}\right\}} \rightarrow 0,
$$

Because of the Lemma 3.2 of Hao and Tang (2008), we know

$$
e^{-h\left(x_{i} / B_{1}\right)} E\left(e^{h N_{i}(T)}\right) \rightarrow 0 .
$$

Hence, for $x_{i}>0$, we have

$$
\begin{aligned}
& \lim _{N \rightarrow \infty} \sup _{t \in(0, T]} \frac{I_{2}\left(x_{i}, t, N\right)}{\int_{0}^{T} P\left(Y_{i} e^{-\delta\left(s+D_{i}\right)} I_{\left\{s+D_{i} \leq T, D_{i} \leq C_{i}\right\}}>x_{i}\right) d m_{i}(s)} \\
& =\lim _{N \rightarrow \infty} \sup _{t \in(0, T]} \frac{I_{2}\left(x_{i}, t, N\right)}{\sum_{\ell=1}^{K} p_{\ell} \int_{0}^{T} \int_{0}^{\alpha_{\ell} \wedge(T-s)} \bar{F}\left(e^{\delta(s+y)} x_{i}\right) d H_{i}(y) d m_{i}(s)} \\
& =0 .
\end{aligned}
$$

For $I_{1}\left(x_{i}, t, N\right)$. Because of the Lemma 1 , for $t \in(0, T]$, we have

$$
\begin{aligned}
I_{1}\left(x_{i}, t, N\right) & \sim \sum_{n=1}^{N} \sum_{i=1}^{n} P\left(Y_{j}^{(i)} e^{-\delta\left(S_{j}^{(i)}+D_{j}^{(i)}\right)} I_{\left\{S_{j}^{(i)}+D_{j}^{(i)} \leq T, D_{j}^{(i)} \leq C_{j}^{(i)}\right\}}>x_{i}, N_{i}(T)=n\right) \\
& \sim\left(\sum_{n=1}^{\infty} \sum_{j=1}^{n}-\sum_{n=N+1}^{\infty} \sum_{j=1}^{n}\right) P\left(Y_{j}^{(i)} e^{-\delta\left(S_{j}^{(i)}+D_{j}^{(i)}\right)} I_{\left\{S_{j}^{(i)}+D_{j}^{(i)} \leq T, D_{j}^{(i)} \leq C_{j}^{(i)}\right\}}>x_{i}, N_{i}(T)=n\right) \\
& =J_{1}\left(x_{i}, t, N\right)+J_{2}\left(x_{i}, t, N\right) .
\end{aligned}
$$

For $J_{1}\left(x_{i}, t, N\right)$, we obtain

$$
\begin{aligned}
J_{1}\left(x_{i}, t, N\right) & =\sum_{j=1}^{\infty} P\left(Y_{j}^{(i)} e^{-\delta\left(S_{j}^{(i)}+D_{j}^{(i)}\right)} I_{\left\{S_{j}^{(i)}+D_{j}^{(i)} \leq T, D_{j}^{(i)} \leq C_{j}^{(i)}\right\}}>x_{i}, N_{i}(T) \geq j\right) \\
& =\int_{0}^{T} P\left(Y_{i} e^{-\delta\left(s+D_{i}\right)} I_{\left\{s+D_{i} \leq T, D_{i} \leq C_{i}\right\}}>x_{i}\right) d m_{i}(s) \\
& =\sum_{\ell=1}^{K} p_{\ell} \int_{0}^{T} \int_{0}^{\alpha_{\ell} \wedge(T-s)} F\left(e^{\delta(s+y)} x_{i}\right) d H_{i}(y) d m_{i}(s) .
\end{aligned}
$$

For $J_{2}\left(x_{i}, t, N\right)$. Applying the similar method of dealing with $I_{2}\left(x_{i}, t, N\right)$, we have

$$
\begin{aligned}
J_{2}\left(x_{i}, t, N\right) & \leq \sum_{n=N+1}^{\infty} \sum_{j=1}^{n} P\left(Y_{j}^{(i)} e^{-\delta\left(S_{1}^{(i)}+D_{j}^{(i)}\right)} I_{\left\{S_{1}^{(i)}+D_{j}^{(i)} \leq T, D_{j}^{(i)} \leq C_{j}^{(i)}\right\}}>x_{i}, N_{i}(T)=n\right) \\
& \leq \sum_{n=N}^{\infty} \sum_{j=1}^{n+1} P\left(Y_{j}^{(i)} e^{-\delta\left(s+D_{j}^{(i)}\right)} I_{\left\{s+D_{j}^{(i)} \leq T, D_{j}^{(i)} \leq C_{j}^{(i)}\right\}}>x_{i}\right) \cdot P\left(N_{i}(T-s)=n\right) d m_{i}(s) \\
& \leq \int_{0}^{T} P\left(Y_{i} e^{-\delta\left(s+D_{i}\right)} I_{\left\{s+D_{i} \leq T, D_{i} \leq C_{i}\right\}}>x_{i}\right) d m_{i}(s) \cdot \sum_{n=N}^{\infty}(n+1) P\left(N_{i}(T) \geq n\right) .
\end{aligned}
$$


Hence, for $x_{i}>0$,

$$
\begin{aligned}
& \lim _{N \rightarrow \infty} \sup _{t \in(0, T]} \frac{J_{2}\left(x_{i}, t, N\right)}{\int_{0}^{T} P\left(Y_{i} e^{-\delta\left(s+D_{i}\right)} I_{\left\{s+D_{i} \leq T, D_{i} \leq C_{i}\right\}}>x_{i}\right) d m_{i}(s)} \\
& =\lim _{N \rightarrow \infty} \sup _{t \in(0, T]} \frac{J_{2}\left(x_{i}, t, N\right)}{\sum_{\ell=1}^{K} p_{\ell} \int_{0}^{T} \int_{0}^{\alpha_{\ell} \wedge(T-s)} \bar{F}_{i}\left(e^{\delta(s+y)} x_{i}\right) d H_{i}(y) d m_{i}(s)} \\
& =0 .
\end{aligned}
$$

By the relations (A1)-(A5), we obtain

$$
\begin{aligned}
& P\left(\sum_{j=1}^{\infty} Y_{j}^{(i)} e^{-\delta\left(S_{j}^{(i)}+D_{j}^{(i)}\right)} I_{\left\{S_{j}^{(i)}+D_{j}^{(i)} \leq T, D_{j}^{(i)} \leq C_{j}^{(i)}\right\}} I_{\left\{S_{j}^{(i)} \leq T\right\}}>x_{i}\right) \\
& \lesssim \sum_{\ell=1}^{K} p_{\ell} \int_{0}^{T} \int_{0}^{\alpha_{\ell} \wedge(T-s)} \bar{F}_{i}\left(e^{\delta(s+y)} x_{i}\right) d H_{i}(y) d m_{i}(s) .
\end{aligned}
$$

Next, we cope with the lower bound of (6).

For $m=0,1,2, \cdots$, we write $\Delta_{m}=\sum_{j=1}^{\infty} Y_{j}^{(i)} e^{-\delta\left(S_{j}^{(i)}+D_{j}^{(i)}\right)} I_{\left\{S_{j}^{(i)}+D_{j}^{(i)} \leq T, D_{j}^{(i)} \leq C_{j}^{(i)}\right\}} I_{\left\{S_{j}^{(i)} \leq T\right\}}$. According to the similar method of Tang and Tsitsiashvili (2004), for all integer $m$ such that $\sum_{j=m+1}^{\infty} j^{-2}<1$, we have

$$
\begin{aligned}
P\left(\Delta_{m}>x_{i}\right) & \leq P\left(\sum_{j=m+1}^{\infty} Y_{j}^{(i)} e^{-\delta\left(S_{j}^{(i)}+D_{j}^{(i)}\right)} I_{\left\{S_{j}^{(i)}+D_{j}^{(i)} \leq T, D_{j}^{(i)} \leq C_{j}^{(i)}\right\}} I_{\left\{S_{j}^{(i)} \leq T\right\}}>\sum_{j=m+1}^{\infty} \frac{x_{i}}{n^{2}}\right) \\
& \leq P\left(\bigcup_{j=m+1}^{\infty}\left(Y_{j}^{(i)} e^{-\delta\left(S_{j}^{(i)}+D_{j}^{(i)}\right)} I_{\left\{S_{j}^{(i)}+D_{j}^{(i)} \leq T, D_{j}^{(i)} \leq C_{j}^{(i)}\right\}\left\{S_{j}^{(i)} \leq T\right\}}>\frac{x_{i}}{n^{2}}\right)\right) \\
& \leq \sum_{j=m+1}^{\infty} P\left(Y_{j}^{(i)} e^{-\delta\left(S_{j}^{(i)}+D_{j}^{(i)}\right)}>\frac{x_{i}}{n^{2}}\right) .
\end{aligned}
$$

Because of the Lemma 2, there exist $0<\alpha<J_{F}^{-} \leq J_{F}^{+}<\infty, A_{i}$ and $B_{i}, i=1,2$ satisfying the relations (4) and (5). The events are written as $A_{1}\left(j, x_{i}\right)=\left(j^{-2} e^{\delta\left(S_{j}^{(i)}+D_{j}^{(i)}\right)} \leq B_{2} / x_{i}\right)$, $A_{2}\left(j, x_{i}\right)=\left(B_{2} / x_{i}<j^{-2} e^{\delta\left(S_{j}^{(i)}+D_{j}^{(i)}\right)} \leq 1\right)$ and $A_{3}\left(j, x_{i}\right)=\left(j^{-2} e^{\delta\left(S_{j}^{(i)}+D_{j}^{(i)}\right)}>1\right)$. Hence, the relation (A7) is written as

$$
\begin{aligned}
P\left(\Delta_{m}>x_{i}\right) & \leq \sum_{j=m+1}^{\infty} P\left(Y_{j}^{(i)} e^{-\delta\left(S_{j}^{(i)}+D_{j}^{(i)}\right)}>\frac{x_{i}}{n^{2}}\right) \\
& =\sum_{k=1}^{3} \sum_{j=m+1}^{\infty} P\left(Y_{j}^{(i)} e^{-\delta\left(S_{j}^{(i)}+D_{j}^{(i)}\right)}>\frac{x_{i}}{n^{2}}, A_{k}\left(j, x_{i}\right)\right) \\
& =\sum_{k=1}^{3} I_{k}\left(m, x_{i}\right) .
\end{aligned}
$$

By Chebyshev's inequality and the Lemma 3 (i), we have

$$
\begin{aligned}
I_{1}\left(m, x_{i}\right) & \leq \sum_{j=m+1}^{\infty} P\left(A_{1}\left(j, x_{i}\right)\right) \leq\left(\frac{x_{i}}{B_{2}}\right)^{-\beta^{\prime}} \sum_{j=1}^{\infty} j^{2 \beta^{\prime}}\left(E\left(e^{-\delta \beta^{\prime} S_{1}^{(i)}}\right)\right)^{j} \\
& =o\left(\bar{F}\left(x_{i}\right)\right) .
\end{aligned}
$$

Because of the relations (4) and (5), for all $x_{i} \geq \max \left\{B_{1}, B_{2}\right\}$, we can obtain

$$
\begin{aligned}
I_{2}\left(m, x_{i}\right) & \leq A_{2} \bar{F}\left(x_{i}\right) \sum_{j=m+1}^{\infty} E\left(j^{2 \beta^{\prime}} e^{-\delta \beta^{\prime} S_{j}^{(i)}} I_{A_{2}\left(j, x_{i}\right)}\right) \\
& \leq A_{2} \bar{F}\left(x_{i}\right) \sum_{j=m+1}^{\infty} j^{2 \beta^{\prime}}\left(E\left(e^{-\delta \beta^{\prime} S_{1}^{(i)}}\right)\right)^{j} .
\end{aligned}
$$


and

$$
\begin{aligned}
I_{3}\left(m, x_{i}\right) & \leq \frac{\bar{F}\left(x_{i}\right)}{A_{1}} \sum_{j=m+1}^{\infty} E\left(j^{2 \alpha^{\prime}} e^{-\delta \alpha^{\prime} S_{j}^{(i)}} I_{A_{3}\left(j, x_{i}\right)}\right) \\
& \leq \frac{\bar{F}\left(x_{i}\right)}{A_{1}} \sum_{j=m+1}^{\infty} j^{2 \alpha^{\prime}}\left(E\left(e^{-\delta \alpha^{\prime} S_{1}^{(i)}}\right)\right)^{j} .
\end{aligned}
$$

Because of $F \in \mathcal{D}$ and $p_{0}>0$, there exists a positive number $M>0$ satisfying for $T \geq T_{0}$,

$$
\begin{aligned}
& Y_{1}^{(i)} e^{-\delta\left(S_{1}^{(i)}+D_{1}^{(i)}\right)} I_{\left\{S_{1}^{(i)}+D_{1}^{(i)} \leq T, D_{1}^{(i)} \leq C_{1}^{(i)}\right\}} I_{\left\{S_{1}^{(i)} \leq T\right\}} \\
& \geq P\left(Y_{1}^{(i)} e^{-\delta\left(S_{1}^{(i)}+D_{1}^{(i)}\right)} I_{\left\{S_{1}^{(i)}+D_{1}^{(i)} \leq T, D_{1}^{(i)} \leq C_{1}^{(i)}\right\}} I_{\left\{S_{1}^{(i)} \leq T\right\}} S_{1}^{(i)} \leq T_{0}\right) \\
& \geq p_{0} P\left(Y_{1}^{(i)} e^{-\delta\left(T_{0}+D_{1}^{(i)}\right)} I_{\left\{S_{1}^{(i)}+D_{1}^{(i)} \leq T, D_{1}^{(i)} \leq C_{1}^{(i)}\right\}} I_{\left\{S_{1}^{(i)} \leq T\right\}}\right) \\
& \geq M \bar{F}\left(x_{i}\right) .
\end{aligned}
$$

By the relations (A8)-(A10), we have

$$
\begin{aligned}
& \lim _{m \rightarrow \infty} \limsup _{x_{i} \rightarrow \infty} \frac{P\left(\Delta_{m}>x_{i}\right)}{\bar{F}\left(x_{i}\right)} \\
& =\lim _{m \rightarrow \infty} \lim \sup _{x_{i} \rightarrow \infty} \sum_{j=m+1}^{\infty} \frac{P\left(Y_{j}^{(i)} e^{-\delta\left(s_{j}^{(i)}+D_{j}^{(i)}\right)}>\frac{x_{i}}{n^{2}}\right)}{\bar{F}\left(x_{i}\right)}=0 .
\end{aligned}
$$

For $\varepsilon>0$, by the relations (A11) and (A12), there exist integer $n_{0}>0$ and enough large number $x_{i}$. Then we have

$$
\begin{aligned}
& P\left(\sum_{j=n_{0}+1}^{\infty} Y_{j}^{(i)} e^{-\delta\left(S_{j}^{(i)}+D_{j}^{(i)}\right)} I_{\left\{S_{j}^{(i)}+D_{j}^{(i)} \leq T, D_{j}^{(i)} \leq C_{j}^{(i)}\right\}} I_{\left\{S_{j}^{(i)} \leq T\right\}}>x_{i}\right) \\
& \leq \sum_{j=n_{0}+1}^{\infty} P\left(Y_{j}^{(i)} e^{-\delta\left(S_{j}^{(i)}+D_{j}^{(i)}\right)}>\frac{x_{i}}{n^{2}}\right) \\
& \leq \varepsilon \bar{F}\left(x_{i}\right) \leq \varepsilon P\left(Y_{1}^{(i)} e^{-\delta\left(S_{1}^{(i)}+D_{1}^{(i)}\right)} I_{\left\{S_{1}^{(i)}+D_{1}^{(i)} \leq T, D_{1}^{(i)} \leq C_{1}^{(i)}\right\}\left\{S_{1}^{(i)} \leq T\right\}}\right) .
\end{aligned}
$$

Let the above number $n_{0}$ be fixed. Because of the Lemma 1, the relation (A13), we know

$$
\begin{aligned}
& P\left(\sum_{j=1}^{\infty} Y_{j}^{(i)} e^{-\delta\left(S_{j}^{(i)}+D_{j}^{(i)}\right)} I_{\left\{S_{j}^{(i)}+D_{j}^{(i)} \leq T, D_{j}^{(i)} \leq C_{j}^{(i)}\right\}} I_{\left\{S_{j}^{(i)} \leq T\right\}}>x_{i}\right) \\
& \geq P\left(\sum_{j=1}^{n_{0}} Y_{j}^{(i)} e^{-\delta\left(S_{j}^{(i)}+D_{j}^{(i)}\right)} I_{\left\{S_{j}^{(i)}+D_{j}^{(i)} \leq T, D_{j}^{(i)} \leq C_{j}^{(i)}\right\}\left\{S_{j}^{(i)} \leq T\right\}}>x_{i}\right) \\
& \sim\left(\sum_{j=1}^{\infty}-\sum_{j=n_{0}+1}^{\infty}\right) P\left(Y_{j}^{(i)} e^{-\delta\left(S_{j}^{(i)}+D_{j}^{(i)}\right)} I_{\left\{S_{j}^{(i)}+D_{j}^{(i)} \leq T, D_{j}^{(i)} \leq C_{j}^{(i)}\right\}\left\{S_{j}^{(i)} \leq T\right\}}>x_{i}\right) \\
& \geq(1-\varepsilon) \sum_{j=1}^{\infty} P\left(Y_{j}^{(i)} e^{-\delta\left(S_{j}^{(i)}+D_{j}^{(i)}\right)} I_{\left\{S_{j}^{(i)}+D_{j}^{(i)} \leq T, D_{j}^{(i)} \leq C_{j}^{(i)}\right\}} I_{\left\{S_{j}^{(i)} \leq T\right\}}>x_{i}\right) .
\end{aligned}
$$

Combining (A6) with (A14) and arbitrariness of $\varepsilon$, we prove the Lemma 4 (1).

(2) Because the inter-arrival times $\left\{\theta_{j}^{(i)}, j \geq 1\right\}$ are $W L O D$ random variables satisfying (9) for some $\epsilon_{i 0}>0$, depending on $F_{i}$ and $G_{i}, i=1,2$, by (11) and (12) of Block et al. (1982), it is clear that

$$
E e^{-\delta \beta^{\prime} S_{n}^{(i)}} \leq\left(E e^{-\delta \beta^{\prime} S_{1}^{(i)}}\right)^{n}
$$

holds for any $\beta^{\prime}>0$ and $n \geq 1$. Hence, we know the relations (A8)-(A10) still hold. Along with the similar proof of Lemma 4 (1), it is easy to prove Lemma 4 (2).

\section{References}

Bingham, Nicholas H., Charles M. Goldie, and Jef L. Teugels. 1987. Regular Variation. Cambridge: Cambridge University Press. 
Block, Henry W., Thomas H. Savits, and Moshe Shaked. 1982. Some concepts of negative dependence. The Annals of Probability 10: 765-72. [CrossRef]

Chan, Wai-Sum, Hailiang Yang, and Lianzeng Zhang. 2003. Some results on ruin probabilities in a two-dimensional risk model. Insurance: Mathematics and Economics 32: 345-58. [CrossRef]

Cline, Daren B. H., and Gennady Samorodnitsky. 1994. Subexponentiality of the product of independent random variables. Stochastic Processes and Their Applications 49: 75-98. [CrossRef]

Embrechts, Paul, Claudia Kllüppelberg, and Thomas Mikosch. 1997. Modelling Extremal Events for Insurance and Finance. Berlin: Springer.

Ghosh, Malay. 1981. Multivariate negative dependence. Communications in Statistics-Theory and Methods 10: 307-37. [CrossRef]

Hao, Xuemiao, and Qihe Tang. 2008. A uniform asymptotic estimate for discounted aggregate claims with subexponential tails. Insurance: Mathematics and Economics 43: 116-20. [CrossRef]

Li, Jinzhu. 2013. On pairwise quasi-asymptotically independent random variables and their applications. Statistics and Probability Letters 83: 2081-87. [CrossRef]

Li, Zehui, and Xinbing Kong. 2007. A new risk model based on policy entrance process and its weak convergence properties. Applied Stochastic Models in Business and Industry 23: 235-46. [CrossRef]

Li, Zehui, Jinxia Zhu, and Feng Chen. 2005. Study of a risk model based on the entrance processes. Statistic and Probability Letters 72: 1-10. [CrossRef]

Li, Junhai, Zaiming Liu, and Qihe Tang. 2007. On the ruin probabilities of a bidimensional perturbed risk model. Insurance: Mathematics and Economics 41: 185-95. [CrossRef]

Liu, Rongfei, and Dingcheng Wang. 2016. The ruin probabilities of a discrete-time risk model with dependent insurance and financial risks. Journal of Mathematical Analysis and Applications 444: 80-94. [CrossRef]

Liu, Xijun, Qingwu Gao, and Yuebao Wang. 2012. A note on a dependent risk model with constant interest rate. Statistics and Probability Letters 82: 707-12. [CrossRef]

Tang, Qihe, and Gurami Tsitsiashvili. 2003. Precise estimates for the ruin probability in finite horizon in a discrete-time model with heavy-tailed insurance and financial risks. Stochastic Processes and their Applications 108: 299-325. [CrossRef]

Tang, Qihe, and Gurami Tsitsiashvili. 2004. Finite-and infinite-time ruin probabilities in the presence of stochastic returns on investments. Advances in Applied Probability 36: 1278-99. [CrossRef]

Wang, Kaiyong, Yuebao Wang, and Qingwu Gao. 2013. Uniform asymptotics for the finite-time ruin probability of a dependent risk model with a constant interest rate. Methodology and Computing in Applied Probability 15: 109-24. [CrossRef]

Xiao, Hongmin, and Jiashan Tang. 2009. Asymptotic Ruin Probabilities of an Entrance Processes Based Risk Model with Interest Force and Regularly Varying Claims. Chinese Journal of Engineering Mathematics 26: 1126-32.

Xiao, Hongmin, and Lin Xie. 2018. Asymptotic ruin probabilities of a dependent renewal risk model based on entrance processes with constant interest rate. Communications in Statistics-Theory and Methods 47: 5396-417.

Xiao, Hongmin, Zehui Li, and Weiwei Liu. 2008. The limit behavior of a risk model based on entrance processes. Computers and Mathematics with Applications 56: 1434-40. [CrossRef]

Xiao, Hong Min, Ze Hui Li, and Wei Wei Liu. 2013. The finite time ruin probability of a new risk model based on entrance process. Communications in Statistics-Theory and Methods 42: 336-45. [CrossRef]

Yang, Yang, and Yuebao Wang. 2010. Asymptotics for ruin probability of some negatively dependent risk models with a constant interest rate and dominatedly-varying-tailed claims. Statistics and Probability Letters 80: 143-54. [CrossRef]

Zhang, Yuanyuan, and Wensheng Wang. 2012. Ruin probabilities of a bidimensional risk model with investment. Statistics and Probability Letters 82: 130-38. [CrossRef]

(C) 2018 by the authors. Licensee MDPI, Basel, Switzerland. This article is an open access article distributed under the terms and conditions of the Creative Commons Attribution (CC BY) license (http:/ / creativecommons.org/licenses/by/4.0/). 